\title{
Observation of Hypersensitivity Phenomena in E-Coli Cells after Irradiation by Alpha Particles
}

\author{
Faika A. Azooz Hassan M. Al-Taa'i \\ Department of Physics \\ College of Education \\ Mosul University
}

\author{
Khalid D. Ahmad \\ Department of Biology \\ College of Education \\ Mosul University
}

(Received 25/6/2008 ; Accepted 16/3/2009)

\begin{abstract}
In order to study the hypersensitivity phenomena in single strand cells, E-Coli bacteria cultures were irradiated by alpha particles emitted from $\mathrm{Am}^{241}$ point source. The cells showed hypersensitivity at 0.05 Gy. Survival curves models were fitted to the data. The recent hypersensitivity models fitted the data much better than the old conventional models. Keywords: E-coli, $\alpha$-particles, hypersensitivity, survival models

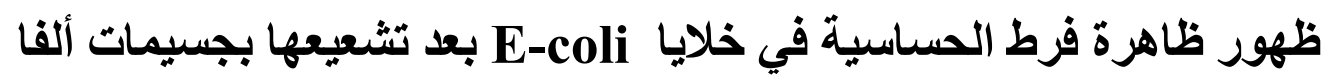

\section{الماضخص}

لغرض درلسة ظاهرة فرط المسلسية في خلايا وحيلة القضيب ، مم تشعبع مستعمرات خلايا E-coli

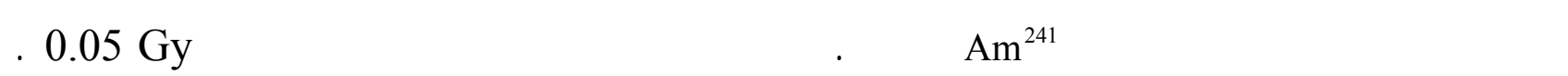
لجريت عملية توافق لمنحنيت النجة. النماذج الحيثة فرط الحسلسية توافتت مع النتائج بشكل أفضل بكثير من النماذج القليدية القنيمة مفتاح الكاملت : أي -كولاي، جسيملت الفا، فرط الهسلسية، منحنيلت النجاة

\section{INTRODUCTION}

Low-dose irradiation is taking a particular importance nowadays, especially because of the observation of the hypersensitivity phenomena in which the cells gains some resistance after irradiation to about $0.5 \mathrm{~Gy}$, where the usual nearly exponential shape of the survival curve, represented by the survival versus dose in Gy, starts to increase at about 0.5 Gy to a higher survival as the dose increases before falling down in a usual manner. This phenomena has been observed in conventional irradiation experiments and in micro beam experiments (Marples et al., 1994, Schettino et al., 2001, Bohrnsen et.al 2002, Linda. 2002b, , Tsoulou et al., 2002, Xiao et al., 2006). Hence, the traditional survival curves models such as the Single Hit Single Target (SHST), and the Molecular model represented by the linear quadratic equation (LQ), both are described by equations below, are no more useful to describe the low dose data:
\end{abstract}


The SHST model is a simple exponential equation (Alpen, 1990) :

$$
S=\exp (-\alpha D)
$$

Where $\mathrm{S}$ is the survival, $\mathrm{D}$ is the dose, which has the same definition in all models and $\alpha$ is the damage factor.

And a quadratic term is added in the LQ equation as (Alpen, 1990):

$$
S=\exp \left(-\alpha D-\beta D^{2}\right)
$$

Where $\alpha$ and $\beta$ are the fitting parameters

The LQ model has been modified by (Joiner and Johns, 1988) to be known as the Inducible Repair model (IR) which is assumed to be applicable to survival curves which show hypersensitivity phenomena as well as those which do not show it, The equation that describe the IR model is given by (Dasu and Denekamp, 2000):

$$
S=\exp \left\{-\alpha_{\mathrm{R}}\left[1+(I R R-1) e^{-\mathrm{D} / \mathrm{Dc}}\right] D-\beta D^{2}\right\}
$$

Where IRR $=\alpha_{S} / \alpha_{R}$

$\alpha_{S}$ is the steep initial slope at very low doses to describe the hypersensitivity region. It changes exponentially as the damage inflicted leads to the induction of more efficient repair, and transformed to a more resistant, shallower slope of the shoulder region after repair has fully induced (characterized by $\alpha$ in the LQ classical model, and now called $\alpha_{R}$ ). The quadratic $\beta$ term takes over at progressively higher doses.

$\mathrm{D}_{\mathrm{C}}$ is the dose at which $63 \%$ (i.e. 1-1/e) of the transition has occurred.

The IR model is so successful in describing survival curves in the low-dose region, however, it describes the high dose region poorly, and a new statistical model, the Repairable Conditionally Repairable model (RCR) was predicted by Linda Persson, in which the survival is given by(Linda, 2002a):

$$
S=\mathrm{e}^{\mathrm{aD}}+b D e^{-\mathrm{cD}}
$$

$\mathrm{a}, \mathrm{b}$ and, $\mathrm{c}$ are fitting parameters

This model is able to describe survival curves at low and high dose reasonably well. In this study, single strand E-Coli bacteria were irradiated by alpha particles emitted from a point source in order to see if they show a hypersensitivity to radiation. Very low doses were used for irradiation $(<0.1 \mathrm{~Gy})$, the survival curve obtained has a shape similar to those which show hypersensitivity in mammalian cells but the inflection point occurs at $0.05 \mathrm{~Gy}$ instead of 0.5 Gy as in mammalian cells. 


\section{Method}

E-coli bacteria were isolated from human colon and cultured for 24 hours at $37{ }^{\circ} \mathrm{C}$ in Agar-Agar medium. Diluted suspensions were prepared using Nutren-Broth, and a point $\mathrm{Am}^{241}$ source which emits alpha particles of energy $5.485 \mathrm{MeV}$ was used for irradiation (Al-Taii , 2006)The source was placed at $2 \mathrm{~cm}$ distance from the Petri dish. The dose rate at the dish surface was calculated according to the relation (Tait, 1980):

$$
\dot{\mathrm{D}}(\mathrm{Gy} / \mathrm{sec})=\Phi_{\mathrm{o}} \times 1.602 \times 10^{-10}(\mathrm{dT} / \rho \mathrm{dX})
$$

Where $\Phi_{\mathrm{o}}$ is the flux in units of particles $/ \mathrm{cm}^{2}$.sec

$\rho$ is the air density and equals to $0.00129 \mathrm{~g} / \mathrm{cm}^{3}$

$\mathrm{dT} / \mathrm{dX}$ is the linear energy transfer in $\mathrm{KeV} / \mu \mathrm{m}$ units

The energy $E_{\alpha}$ at 2-cm distance from the source was calculated using the energy range relationship(Mahesh and Mustafa, 1976):

$\mathrm{E}_{\alpha}^{(\mathrm{x})}=\mathrm{E}_{\alpha}^{(0)}\left(1-\frac{\mathrm{x}}{\mathrm{R}}\right)^{\frac{2}{3}}$

$\mathrm{E}_{\alpha}^{(\mathrm{x})}$ is the initial alpha particle energy

$\mathrm{E}_{\alpha}^{(0)}$ is the energy after traveling a distance $\mathrm{x}$

$\mathrm{R}$ is the range which is given by

$\mathrm{R}=(0.005+0.285) \mathrm{E}^{3 / 2}$

The range was found to be $4.007 \mathrm{~cm}$ at which $\mathrm{E}_{\alpha}^{(\mathrm{x})}=3.45 \mathrm{MeV}$

This value of $E_{\alpha}^{(x)}$ was used in SRIM2003 program (Ziegler, 2003) to calculate $d T / d X$ which is found to be $97.7 \mathrm{KeV} / \mu \mathrm{m}$

To calculate $\Phi_{\mathrm{o}}$, assume that $\mathrm{N}$ is the number of particles emitted over a $4 \Pi$ solid angle per second. This simply represents the activity of the source. Hence, the number of particles at 2 $\mathrm{cm}$ distance from the source in all directions will be

$\Phi_{\mathrm{o}}=\mathrm{N} / 4 \pi \mathrm{r}^{2}$

An ${ }^{241} \mathrm{Am}$ source of activity $1 \mu \mathrm{Ci}$ was used in this experiment, This gives $\Phi_{\mathrm{O}}=763.5$ Particles $/ \mathrm{cm}^{2} . \mathrm{sec}$

Substituting the values of $\Phi_{\mathrm{O}}$ and $\mathrm{dT} / \mathrm{dX}$ in equation (5), we can calculate the dose rate at 2cm distance.

Assuming 10\% loses due to backscatter, geometrical arrangements and efficiency of the measurements. The dose rate was found to be $1 * 10^{-4} \mathrm{~Gy} / \mathrm{sec}$.

After irradiation, carried out in medium at room temperature, the samples were washed, diluted with fresh medium and visible colonies were scored for survival evaluation using the method of point counting where each dark point represents a colony ( see figure 1) - In every experiment two independent samples were irradiated for a given dose with a control Petri dish for each. 


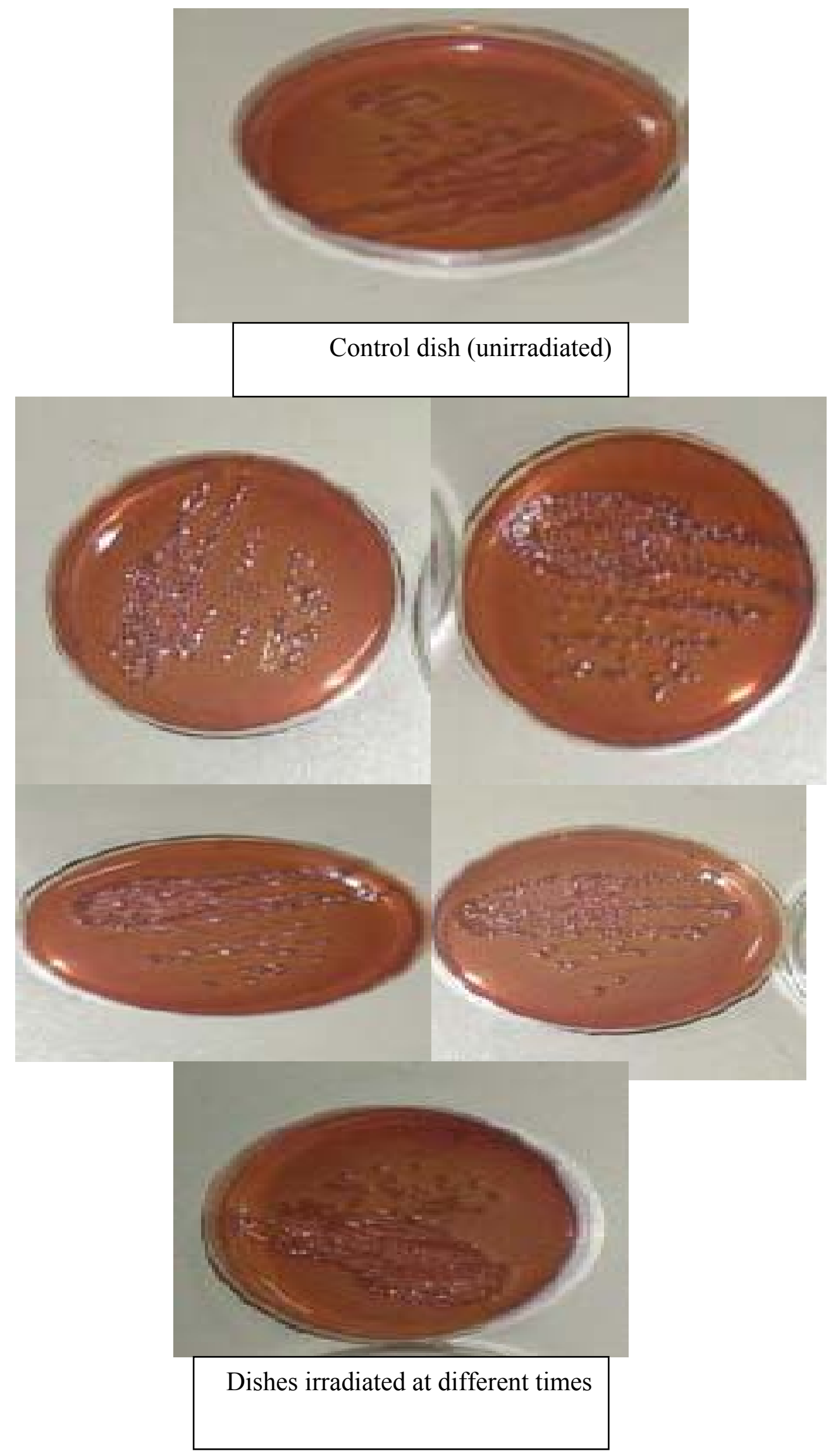

Fig. 1: Control and irradiated Petri dishes. The dark points indicate colony formation 


\section{RESULTS AND DISCUSSION}

The survival versus dose for irradiation of E-Coli cells by $3.45 \mathrm{MeV}$ alpha particles is shown in figure (2). It is clear that, the curve does not take the usual semi exponential shape of the survival curve. The survival falls down to 0.15 at $0.05 \mathrm{~Gy}$ and starts to increase to about 0.25 before it starts to decrease again slowly. This implies that the cells have gained some resistance against radiation similar to what have been seen in mammalian cells hypersensitivity which occurs at about 0.5 Gy.

To test for hypersensitivity phenomena we used four survival curves models; These are: the SHST model and the LQ model, which both are used to describe data of usual survival curves which show no peculiar structure, represented by equation 1 and 2 respectively, and the IR model and the RCR model which were predicted to describe the hypersensitivity phenomena and represented by equations 3 and 4 respectively,

The fit of these models to the data is shown in figure (3). To test the goodness of fit, four statistical quantities have been examined; These are ; the Sum of Squares due to Error (SSE) which should be close to zero, the R-square (RSQ) value which should be close to 1 or it is better to use the Adjusted R-square (ADJ) value which takes into account the number of degrees of freedom $(v)$ where $v=n-m, n$ is the number of fitted points and $m$ is the number of fitted parameters, also the Root Mean square Error (RMSE) should have a value close to zero (see Mat-Lab help for details). As clearly seen from figure 3, the SHST model and the LQ model do not fit the data in the 0.05 Gy region,. and as seen from table 1 , the statistical quantities that describe the goodness of fit for these models are far from acceptable, although we have tried our best to improve them. We have learned from our experience with Matlab fitting programs that when SSE is greater than 0.1 or ADJ is less than 0.9 the fit is bad. However, the IR model and the RCR model describe the shape of the curve much better, where SSE for these two models is less than 0.1 and the ADJ, although it is not very close to 1 , but a value of about 0.9 is acceptable. This means a hypersensitivity phenomena is present in single strand cells as E-Coli bacteria and not restricted to double stranded cells as it was thought before.

It is difficult to compare our results with previous data since almost all the hypersensitivity measurements have been carried out on mammalian cells (i.e. double stranded) while E-coli bacteria cells are simple single stranded cells. But, in general, the damage factor which describe the cell kill at the initial slope of the survival curve is about the same for a given type of radiation at a given energy. This factor is represented by $\alpha$ in the SHST model and the LQ model and by $\alpha_{R}$ in the IR model and as seen from table 1 it has similar values in these models. 
Faika A. Azooz et al.

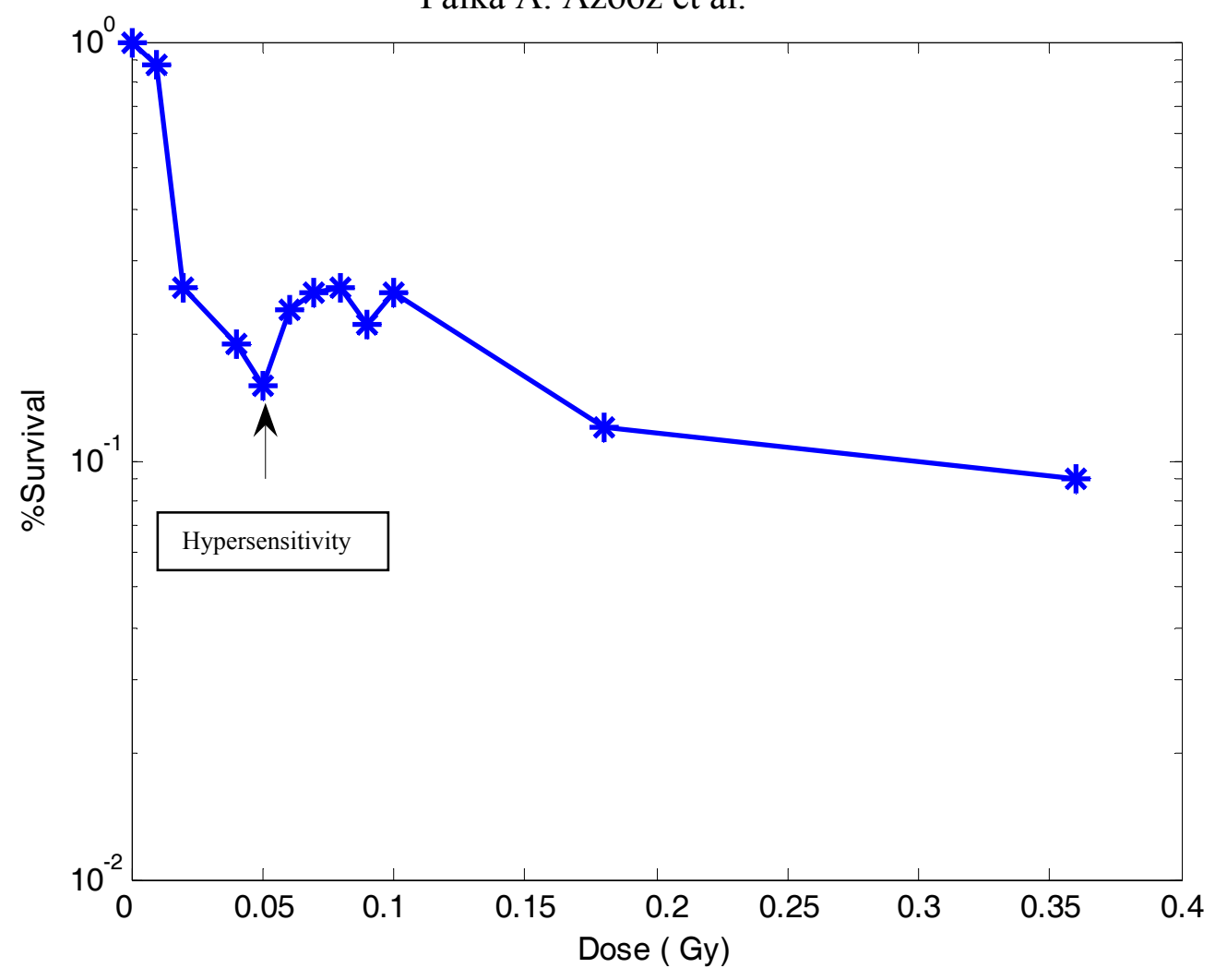

Fig. 2: Experimental data points for the survival fraction of E-coli cells as a function of Dose

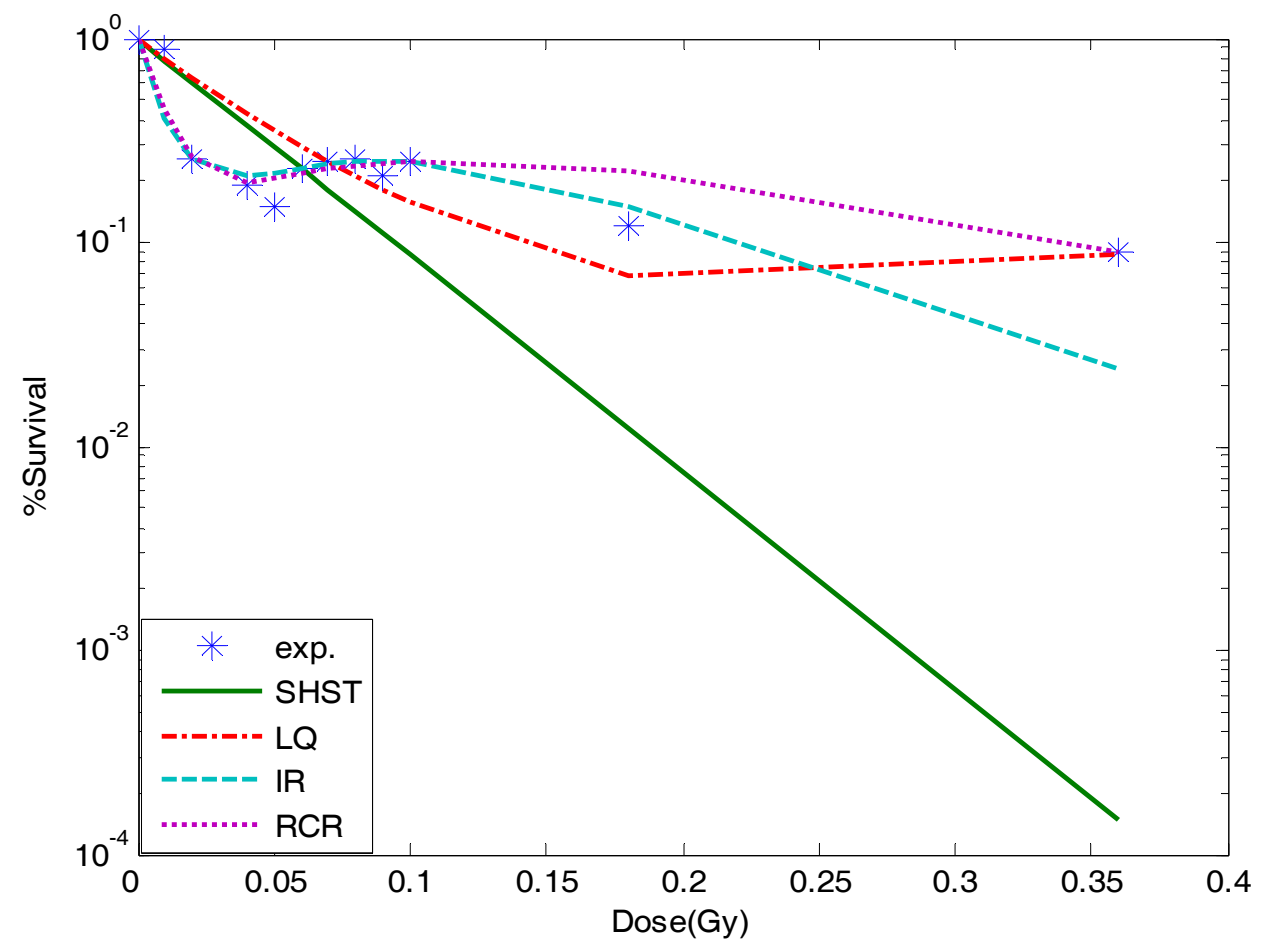

Fig.3: The survival fraction of E-coli cells as a function of dose Fitted to SHST, LQ, IR, and RCR equations. 
Table 1: Biophysical and Statistical parameters obtained from the fit.

\begin{tabular}{|c|c|c|c|c|c|c|}
\hline & & \multicolumn{3}{|c|}{$\mathrm{E}=3.45 \mathrm{MeV}$} & \multicolumn{2}{|c|}{ LET $=97.7(\mathrm{KeV} / \mu \mathrm{m})$} \\
\hline & & & SSE & RSQ & ADJ & RMSE \\
\hline SHST & $\alpha$ & $\begin{array}{c}2.45 \\
(+0.92,-0.92)\end{array}$ & 0.124 & 0.765 & 0.733 & 0.0538 \\
\hline \multirow{2}{*}{$\mathbf{L Q}$} & $\alpha$ & $\begin{array}{c}2.24 \\
(+0.87,-0.87)\end{array}$ & \multirow{2}{*}{0.077} & \multirow{2}{*}{0.797} & \multirow{2}{*}{0.782} & \multirow{2}{*}{0.0752} \\
\hline & $\beta$ & $\begin{array}{c}-0.4523 \\
(+0.098,-0.098)\end{array}$ & & & & \\
\hline \multirow{3}{*}{$\mathbf{R C R}$} & $\mathrm{a}$ & $\begin{array}{c}9.205 \\
(+2.87,-3.2)\end{array}$ & \multirow{3}{*}{0.039} & \multirow{3}{*}{0.9502} & \multirow{3}{*}{0.9392} & \multirow{3}{*}{0.0725} \\
\hline & $\mathrm{b}$ & $\begin{array}{c}0.58 \\
(+0.115,-0.21)\end{array}$ & & & & \\
\hline & $\mathrm{c}$ & $\begin{array}{c}0.8855 \\
(+0.258,-0.198)\end{array}$ & & & & \\
\hline \multirow{4}{*}{ IR } & $\alpha_{\mathrm{R}}$ & $\begin{array}{c}2.39 \\
(+0.78,-0.69)\end{array}$ & \multirow{4}{*}{0.076} & \multirow{4}{*}{0.91} & \multirow{4}{*}{0.905} & \multirow{4}{*}{0.082} \\
\hline & $\alpha_{S}$ & $\begin{array}{c}3.79 \\
\text { (fixed at bound) }\end{array}$ & & & & \\
\hline & $\beta$ & $\begin{array}{c}0.0 \\
\text { (fixed at bound) }\end{array}$ & & & & \\
\hline & $\mathrm{D}_{\mathrm{c}}$ & $\begin{array}{c}0.033 \\
\text { (fixed at bound) }\end{array}$ & & & & \\
\hline
\end{tabular}

\section{REFERENCES}

Alpen, E. L., 1990. Radiation Biophysics., Prentice- Hall International, Inc.

Al-Taii, H., 2006. A Comparison Between Theoretical Models That Describe Survival Curves for Cell Irradiation, M.Sc. Thesis, Mosul Univ.

Bohrnsen, G ; Weber. K.J and Scholz.M., 2002. Low Dose Hypersensitivity and Resistance of V79 Cells After Charge Particle Irradiation Using $100 \mathrm{MeV} / \mathrm{u}$ Carbon ions, Radiat. Prot. Dosi., Vol. 99, N.1-4, pp. 255 -256.

Dasu, A. and Denekamp J., 2000. Inducible Repair and Intrinsic Radiosensitivity, Radiat. Res, Vol 153, pp.279-288.

Joiner, M.C. and Johns, H., 1988. Renal Damage in the Mouse: The Response to Very Small Doses Per Fraction, Radiat. Res., Vol. 114, pp. 385-398 .

Linda, M. Persson; Bengt K. Lind; Ingegrd Hedlöf; Margareta R. Edgren. and Anders Brahme., 2002b. Low-Dose Hypersensitivity in Human Colon and Small Cell Lung Cancer Cells. Email: Linda@,radfys.ks.se. Paper II, Radiation Biology. 
Linda, M.; Persson, L.M.; Edgren, M.R., Hedlöf and I.andbrahame, A, 2002a. Repairable Conditionally Repairable Damage Model Based on Dual Poisson Processes. Submitted to Radiation Res., Paper(I).

Mahesh, K. and Mustafa S., 1976. Nuclear Radiation Detector and Experiment. Mosul University Press.

Marples, B.; Skov, K.A. and Joiner, M.C.,1994. The Effect of Oxygen on Low-Dose Hypersensitivity and Increased Radioresistnce in Chinese Hamster V79-379Acells. Radiat. Res., Vol. 138, pp.17-20, Abstract.

Schettino, G.; Folkard, M.; Prise, K.M.; Vojnovic, B.; Bowey, A.G. and Michael, B.D., 2001. Low-Dose Hypersensitivity in Chinese Hamster V79 Cells Target with Counted Protons Using a Charged-Particle Microbeam, Radiat. Res., Vol. 156, pp.526-534.

Tait, W.H., 1980. Radiation Detection, Butterworths.

Tsoulou, E.; Baggio, L.; Cherubini. R. and Kalfas, C.A, 2002. Radiosensitivity of V79 Cells after ALPHA Particle Radiation at Low Dose , Radiat. Prot. Dosi., Vol .99, No. 1-4, pp.237- 240.

Xiao, dong Jin; Qiang Li; Wen-jian, Li; Wang, Jn-fang; Chuan-ling Guo and Hao, Ji. Fang, 2006. The Hyper- Radiosensitivity Effect of Human Hepatoma SMMC-7721 Cells Exposed to Low Dose $\gamma$-Rays

Ziegler, J., SRIM, 2003. program, The Stopping and Ranges of Ions in Matter, http://www.software.ibm.com/ad/apl/apl2.html 